\title{
Towards 3D ultrasound guided needle steering robust to uncertainties, noise and tissue heterogeneity
}

\author{
Guillaume Lapouge ${ }^{1,2, *}$, Philippe Poignet $^{1}$, Jocelyne Troccaz ${ }^{2}$
}

\begin{abstract}
This paper presents a new solution for 3D steering of flexible needles guided by 3D B-mode ultrasound imaging. It aims to realize a robust steering, by accounting for uncertainties, noise and tissue heterogeneities, while limiting tissue-related disturbances. The proposed solution features interconnected state observer, automatic needle tip segmentation and path planning algorithms. Measurement quality, state uncertainties and tissue heterogeneity are considered for robust needle steering with helical paths of variable curvature. Fast replanning allows for adaptability to unexpected disturbances. An experimental validation has been done through 62 insertions of 24 Gauge bevel-tip nitinol needles in various tissue. Results are promising, characterized by mean targeting errors of less than $1 \mathrm{~mm}$ in homogeneous phantoms, $1.5 \pm 0.9 \mathrm{~mm}$ in heterogeneous phantoms and $1.7 \pm 0.8 \mathrm{~mm}$ in ex-vivo tissue. This new approach is a step towards a precise and robust patient-specific gesture.
\end{abstract}

Index Terms-path planning, needle steering, 3D ultrasound imaging

\section{INTRODUCTION}

\section{State of the Art for Image-based Needle Steering}

The context of this paper is percutaneous surgical procedures in which a physician inserts a needle into biological tissue to realize biopsies, drug delivery, brachytherapy etc. To increase the precision and the capabilities of such operations, robotic needle steering has been introduced with the added value of obstacle avoidance, target tracking, complex path computation and an increased targeting precision. There are several ways to guide a flexible needle into soft tissue. In this paper, we consider beveled tip needles. The asymmetry of the tip imbalances the forces applied to it and causes natural deflection in a privileged direction during the insertion. When rotated around its main axis, the needle deflection direction changes, allowing 3D control of this nonholonomic system.

In this paper, we will focus on computing a complex path to reach a target. More information on control objectives and issues in needle steering can be found in [1].

To begin with, many algorithms for needle steering are validated through simulation. Tracking for $2 \mathrm{D}$ steering has been developed in a simulated environment with stochastic motion roadmap in [2] to account for uncertainties. It has been extended to 3D simulated steering in [3]. In [4] both modeling

This work was partly supported by the Investissements d'Avenir programme (Labex CAMI) under reference ANR-11-LABX-0004.

${ }^{1}$ Univ. Montpellier, CNRS, LIRMM, F-34090 Montpellier, France

${ }^{2}$ Univ. Grenoble Alpes, CNRS, Grenoble INP, TIMC-IMAG, F-38000 Grenoble, France

* Corresponding author, guillaume.lapouge@univ-grenoble-alpes.fr and measurement uncertainties are taken into account. [5] completes this work with fast replanning for intra-operative adaptation to system uncertainties.

However, steering needles in real objects represents a significant leap in complexity.

First, image-based needle detection proves to be challenging. Indeed, clinically compatible imaging sensors either provide a poor image quality (e.g. ultrasound (US) imaging), or present security and cost constraints (e.g. MRI systems and CT scanners). In spite of its limitations such as artifacts, low resolution and sampling rate [6], ultrasound imaging is the preferred clinical imaging solution for needle insertion. It allows for simple and affordable real-time imaging. However, because the needle is barely visible in ultrasound images, several solutions [7], [8], involve the robotized translation of a $2 \mathrm{D}$ probe (also called 2.5D) so that the needle tip is always in the best detection configuration. Such a translation may not always be clinically feasible. In [9] and [10], 3D ultrasound imaging is used in Doppler mode to detect a vibrated or rotated needle. Yet, the localization precision remains low despite an increased technical complexity (vibrating device, adapted control strategy, etc.). With the exception of [11], [12] and [13], there is, to our knowledge, no 3D B-mode ultrasound guided flexible needle steering.

Furthermore, because of tissue heterogeneity, tissue deformation and modeling uncertainties, the needle does not have a deterministic behavior. For more robustness to such uncertainties, a fast trajectory replanning approach is adopted in many experimental works [14], [15], [13], [16], [17], [18]. Online curvature estimation for the needle kinematic model has also proven to be useful [19], [20] as the observed curvature might vary from the expected one and tissue may be heterogeneous. The curvature of the needle may also be controlled, thus simplifying the steering. To do so, a first approach consists in using an actuated needle. Nonetheless, such a solution decreases the clinical compatibility by requiring a specific instrument. A second approach is to adopt a dutycycling approach and alternate between pure insertions and insertions with high speed rotation [21]. However, in biological tissue, repeated rotations may cause additional issues such as increased needle torsion, tissue winding and trauma [13], [22].

\section{Contribution}

This paper presents a novel 3D needle steering approach specifically designed for robustness to low needle visibility 


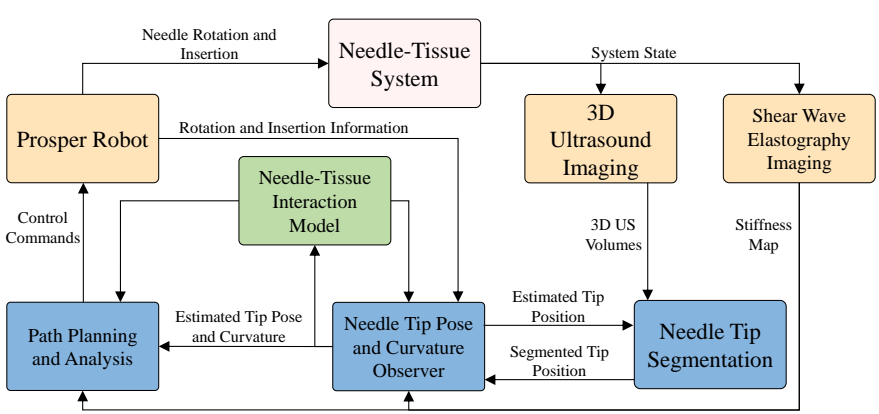

Fig. 1: Overview of the sub-processes of the proposed needle steering solution.

in 3D US volumes and potential disturbances caused by heterogeneous media. The contribution is manifold:

- stiffness map-based path planning with helical paths of variable curvature;

- interconnection of adaptive and asynchronous new methods to achieve robustness (cf. Fig. 1);

- experimental validation with varied materials and needles.

In sections II-B and II-C needle localization methods compatible with 3D B-mode ultrasound imaging and previously validated are quickly introduced.

A new path planning algorithm is detailed in section II-D. This algorithm generates a plan relying not only on pose estimation, but also on curvature estimation and prediction. This allows to significantly reduce tissue trauma and fibers winding around the needle shaft. Indeed, the algorithm computes smooth 3D helical paths with depth-dependent and tissue specific curvature, thus removing the need for duty cycled insertions.

The curvature is estimated both online for the current needle position and offline along planned needle paths through a pre-operative tissue stiffness map, as the tissue may present some heterogeneity. The path planning runs in two steps: fast planning and in-depth path analysis. We propose new metrics for extensive path quality analysis taking into account state estimation uncertainty, variable measurement quality and tissue trauma.

An experimental validation is described in section III. Insertions were made in homogeneous phantoms, heterogeneous phantoms and ex-vivo tissue with significant progress in robustness and precision when compared to our previous results in [13]. Further relevant comparison with the state of the art is also provided in Table III.

\section{Materials And Methods}

\section{A. Overview}

This paper proposes a novel flexible needle steering method compatible with 3D ultrasound B-mode imaging. This method can be subdivided into clearly defined sub-processes (see Fig. 1).

On the hardware side, the Prosper robot developed in [23] inserts a flexible needle into soft tissue (see Fig. 2). The feedback is given by the robot sensors, 3D ultrasound imaging

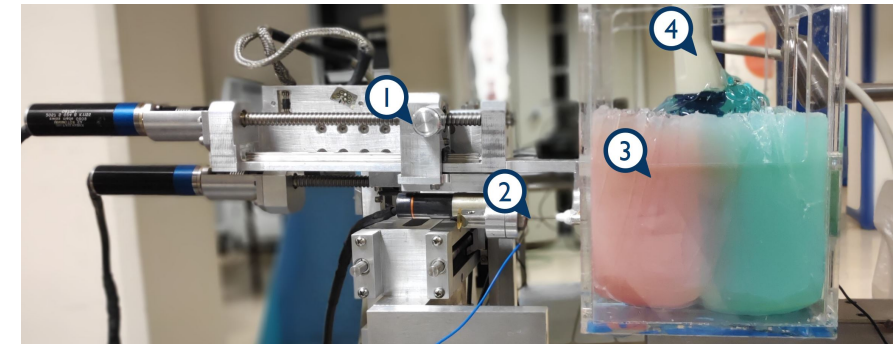

Fig. 2: Experimental setup. Prosper Robot (1); Needle (2); Phantom (3); US probe (4).

and Shear Wave Elastography (SWE) measurements. Detailed information on hardware can be found in section III-A.

The software solution is composed of :

- a needle tip pose and curvature observer (see section II-B),

- a needle tip segmentation algorithm (see section II-C),

- a path planning and control algorithm (see section II-D).

Although each algorithm block has been developed and validated independently, the robustness of the proposed solution is enhanced by the interconnection between the blocks.

\section{B. Tip Pose Modeling and Estimation}

A critical issue in needle steering is the accurate estimation of the needle state.

\section{i) Needle-Tissue Interaction Model}

The needle tip behavior is modeled by a modified unicycle model that we propose. As in [24], when inserted without rotation, the needle tip traces a circular arc of curvature $\kappa$. If rotated by $180^{\circ}$, the needle traces another arc in the opposite direction, not tangent to the first one, with a discontinuity angle $\beta_{\text {cut }}$.

Rotations are expressed using Tait-Bryan angles (see Fig. 3 ). This model is built from the regular unicycle approach introduced in [25]. With the hypothesis that $\beta$ is small, $\beta_{\text {cut }}$ is modeled as a deviation of $\frac{\beta_{\text {cut }}}{2} \cos (-\gamma)$ in yaw and of $\frac{\beta_{c u t}}{2} \sin (-\gamma)$ in pitch of the needle as it is rotated. The virtual wheel of the kinematic motion model is therefore aligned with the direction of insertion $x_{t}$, as illustrated in Fig. 3.

Finally, this model can be seen as an extension of the one presented in [24], that does not limit the needle motion modeling to piecewise circular curves. It should be noted that the model does not strictly represent the motion presented in [24], but that is not noticeable in practice, under the conditions that $\beta$ and $\beta_{\text {cut }}$ are small. The model can be written in the joint state-space as $\dot{\boldsymbol{x}}=\boldsymbol{f}(\boldsymbol{x}, \boldsymbol{u})$ as follows:

$$
\left[\begin{array}{c}
\dot{x} \\
\dot{y} \\
\dot{z} \\
\dot{\alpha} \\
\dot{\beta} \\
\dot{\gamma} \\
\dot{\kappa}
\end{array}\right]=\left[\begin{array}{cc}
\cos \alpha \cos \beta & 0 \\
\sin \alpha \cos \beta & 0 \\
-\sin \beta & 0 \\
\kappa \cos \gamma \sec \beta-\kappa \frac{\beta_{c u t}}{2} \sin \gamma \cos \gamma \tan \beta & -\frac{\beta_{c u t}}{2} \sin \gamma \\
-\kappa \sin \gamma-\kappa \frac{\beta_{c u t}}{2}(\cos \gamma)^{2} \tan \beta & -\frac{\beta_{c u t}}{2} \cos \gamma \\
\kappa \cos \gamma \tan \beta & 1 \\
0 & 0
\end{array}\right]\left[\begin{array}{l}
u_{1} \\
u_{2}
\end{array}\right],
$$




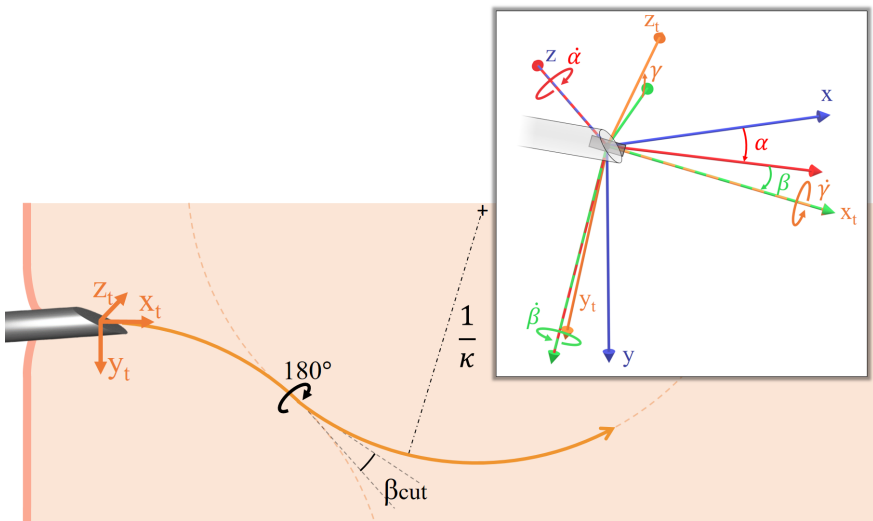

Fig. 3: Modified unicycle kinematic model. When the needle is rotated of $180^{\circ}$, the path bends with the angle $\beta_{c u t}$.

where $x, y, z$ are the Cartesian coordinates of the needle tip in the 3D US volume frame [mm]; $\alpha, \beta$ and $\gamma$ are the yaw, pitch and roll of the needle tip [rad]; $\kappa$ is the curvature of the needle tip trajectory $\left[\mathrm{mm}^{-1}\right]$. The inputs $u_{1}$ and $u_{2}$ correspond respectively to the insertion speed $\left[\mathrm{mm} . \mathrm{s}^{-1}\right]$ and the rotation speed $\left[\mathrm{rad} . \mathrm{s}^{-1}\right.$ ] of the needle tip. $\beta_{c u t}$ is the cutting angle [rad] described in Fig. 3.

\section{ii) Needle Tip Pose and Curvature Observer}

A multi-rate unscented Kalman filter runs asynchronously to take into account measurements coming from :

- a sensor providing the orientation of the needle base;

- the segmentation algorithm detailed in section II-C;

- SWE measurements.

The observer gives a robust estimation of the needle tip pose with a mean error of $0.6 \mathrm{~mm}$ [19]. It also provides an online estimate of the needle curvature $\kappa$. The observer does not constrain the needle motion to successive circular in-plane motions, as it does not rely on circle fitting to estimate the needle path curvature. Therefore, it allows for a greater freedom in the control strategy. More details about the estimation methods are available in [19].

\section{Needle Tip Segmentation}

Because of real-time constraints, processing the entire 3D US volume should be avoided. Considering a follow the leader motion of the needle shaft, the estimated needle tip path provides prior knowledge of the needle position to the segmentation algorithm. The needle segmentation can therefore be focused to a specific region of interest (ROI), defined as a curved tube centered around the estimated needle tip path. Its radius can be set by the user depending on the diameter of the chosen needle shaft (see Fig. 4). At the current estimated tip location, the region of interest radius may be increased for a more flexible tip localization in noisy US volumes at the cost of increased risks to falsely detect artifacts as the needle tip. In the following sections, only data inside the ROI are considered.

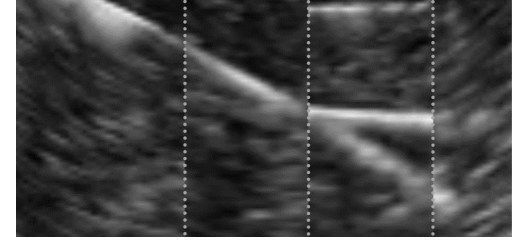

(a) Original 3D US volume. The bright horizontal trace was created by a previous needle insertion.

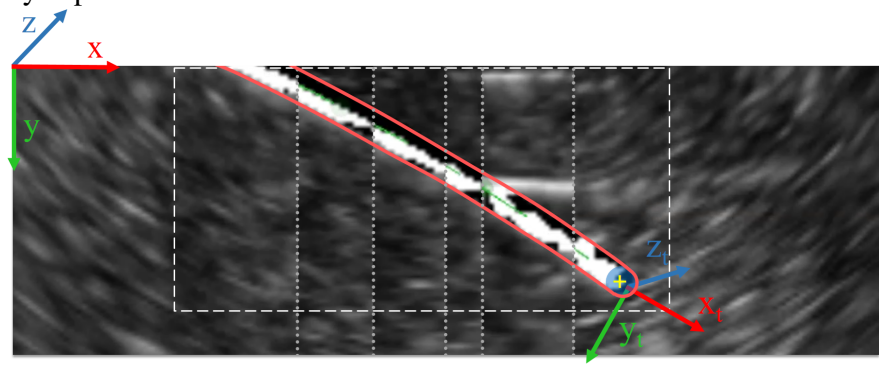

(b) In the tubular region of interest (red tube), white voxels were classified in the needle class, dark ones in the background class. In the blue search area, the tip is segmented (yellow cross).

Fig. 4: Needle tip segmentation in a 3D US volume. The needle in the 3D volume is visualized with a combination of $2 \mathrm{D}$ slices (delimited by dotted lines).

The acquired 3D US volumes are subject to artifacts and noise. It is frequently a challenge to determine which voxels belong to the needle. Furthermore, depending on the observed medium, the histogram of the voxels intensity can vary drastically.

To solve this issue, we propose to use the naive Bayesian classification approach detailed in [26]. The intensity histogram is approximated by a sum of two normal distributions: a needle normal distribution $\mathcal{N}_{n}$ and a background normal distribution $\mathcal{N}_{b}$. The probability distribution parameters are estimated using Expectation-Maximisation. Each voxel of the ROI is then classified as belonging to the needle class if its probability to belong to $\mathcal{N}_{n}$ is superior to its probability to belong to $\mathcal{N}_{b}$.

The needle tip detection is done around the estimated needle tip position i.e. at the tip of the ROI (see Fig. 4). The estimated tip coordinates $\left(x_{0_{\mathcal{F}}}, y_{0_{\mathcal{F}}}, z_{0_{\mathcal{F}}}\right)$, in the image frame $\mathcal{F}$, are provided by the observer described in section II-B.

In its neighborhood, the needle tip is detected as the weighted centroid of the voxels belonging to the needle class. The weighting $w_{p}$ of a voxel $p$ verifies:

$$
w_{p} \propto w_{p_{1}} x_{\mathcal{F}_{t}}-w_{p_{2}}\left(y_{\mathcal{F}}-y_{0_{\mathcal{F}}}\right),
$$

where $w_{p_{1}}, w_{p_{2}} \in \mathbb{R}_{+}^{2} ; x_{\mathcal{F}_{t}}$ is the abscissa of the voxel in the estimated tip frame $\mathcal{F}_{t} ; y_{\mathcal{F}}$ is the ordinate of the voxel in the image frame $\mathcal{F}$.

The term in " $x_{\mathcal{F}_{t}}$ " corresponds to the research of the needle tip in the direction of the insertion, whereas the term " $y_{\mathcal{F}}-$ $y_{0_{\mathcal{F}}}$ " aims at avoiding the bias caused by reverberation artifacts such as comet tail artifacts or guided wave artifacts (see [6] for more details).

The proposed algorithm allows for fully automatized binarisation, without relying on manual thresholding. Although the 
coefficients $w_{p_{1}}$ and $w_{p_{2}}$ are empirically set by the user, this segmentation method has proven to be robust to needle and tissue changes. As it can be seen in Fig. 4, it is also robust to temporary presence of bright spots and traces of previous insertions.

The algorithm is sensitive to the ratio $\frac{w_{p_{1}}}{w_{p_{2}}}$. As the research of the needle tip must be done mainly towards $x_{t}$, this ratio is constrained to $\frac{w_{p_{1}}}{w_{p_{2}}}>1$. To prevent a shift towards the reverberation artifacts, we recommend to tune $2<\frac{w_{p_{1}}}{w_{p_{2}}}<10$.

Robustness to temporary needle loss is ensured by the data fusion that is done by the observer. When no voxel classified in the needle class is found in the tip search area, the segmentation fails. However, using equation (1), the observer still estimates the needle tip position from the inputs $u_{1}, u_{2}$ and other available measurements to recover it whenever it is visible.

More details about the segmentation methods are available in [27].

\section{Path Planning and Analysis}

In an attempt to prevent tissue lesion and fibers winding around the needle observed in [13] and [22], 3D helical paths are preferred over the duty cycling approach described in [21]. The trajectories are therefore done at a constant insertion speed of $0.5 \mathrm{~mm} . \mathrm{s}^{-1}$ and at piecewise constant and slow rotation speed.

Generating such trajectories requires significant computation time. A two step approach is adapted here to achieve fast replanning: fast path planning and in-depth path analysis.

The approach detailed below aims at introducing a generalized robust path planning solution which allows for complex motion and obstacle avoidance. It is robust to the chosen needle and insertion medium, heterogeneity in the tissue and uncertainties both in the measurements and in the state estimation.

\section{i) Reachability-guided RRT}

The proposed path planning is based on an input sampling RRT which considers reachability as in [28].

Based on the model described by (1), this algorithm builds paths from a configuration $\boldsymbol{q}_{\text {init }}$ to a target $\boldsymbol{p}_{\text {goal }}$ while avoiding obstacles (cf. Fig. 5). $\boldsymbol{q}_{\text {init }}$ is taken equal to the needle tip state estimate when the planning starts. A point $\boldsymbol{p}_{\text {rand }}$ is drawn randomly in the working space $\mathcal{W} \subset \mathbb{R}^{3}$ (cf. Fig. 5a). The closest tree node $\boldsymbol{q}_{\text {near }}$ from $\boldsymbol{p}_{\text {rand }}$ is then chosen and the tree is grown from $\boldsymbol{q}_{\text {near }}$ by applying inputs in $U_{\text {new }}$. $U_{\text {new }}=\left[\left(u_{1}, l_{1}\right) ;\left(u_{2}, l_{2}\right) \ldots\left(u_{n}, l_{n}\right)\right]$ where $u_{i} \in\left[u_{\min }, u_{\max }\right]$ and $l_{i} \in\left[l_{\min }, l_{\max }\right]$ are rotation speed and insertion length inputs respectively. To simplify the determination of the reachable space, the unicycle model $\left(\beta_{c u t}=0\right)$ is used for its computation. This approximation can be done safely as it underestimates the reachable space of the needle. Besides, the value of $\beta_{c u t}$ is small, thus reducing the difference between true and approximated reachable spaces. With this hypothesis, the reachable space of the needle in the configuration $\boldsymbol{q}$ is delimited by a solid torus in $\mathbb{R}^{3}$ of radii equal to $\frac{1}{\kappa}$, the needle

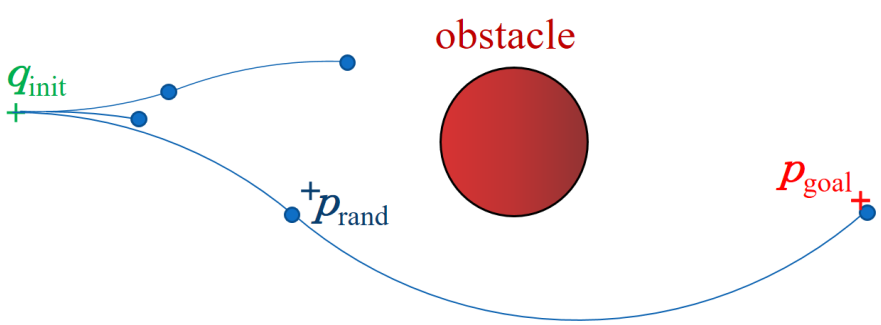

(a) A point $\boldsymbol{p}_{\text {rand }}$ is randomly drawn in $\mathcal{W}$.

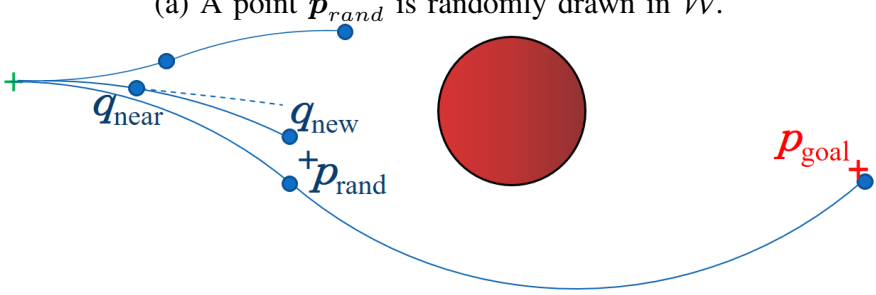

(b) Reachability-guided choice of the closest node, creation of $\boldsymbol{q}_{\text {new }}$ from the best sampled input.

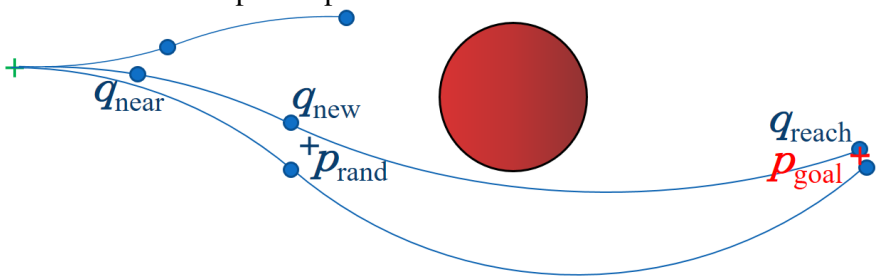

(c) Connection of $\boldsymbol{q}_{\text {new }}$ to the target $\boldsymbol{p}_{\text {goal }}$, creation of $\boldsymbol{q}_{\text {reach }}$.

Fig. 5: Detailed RRT growth. The tree (in blue) must link an initial configuration $\boldsymbol{q}_{\text {init }}$ to the target $\boldsymbol{p}_{\text {goal }}$ while avoiding an obstacle (in red). The figure is plotted with $\beta_{c u t}=0$ for more clarity.

radius of curvature. In the needle tip frame $\mathcal{F}_{t}$, these points have constrained Cartesian coordinates $\left(x_{\mathcal{F}_{t}}, y_{\mathcal{F}_{t}}, z_{\mathcal{F}_{t}}\right)$ so that

$$
x_{\mathcal{F}_{t}} \geq \sqrt{\frac{2}{\kappa} \sqrt{\left(y_{\mathcal{F}_{t}}^{2}+z_{\mathcal{F}_{t}}^{2}\right)}-y_{\mathcal{F}_{t}}^{2}-z_{\mathcal{F}_{t}}^{2} .}
$$

The euclidean norm does not take into account the nonholonomy of the system. In other words, the closest node it defines does not guaranty the reachability of $\boldsymbol{p}_{\text {rand }}$. Therefore, the research of the closest node is reduced to the subset of nodes from which $\boldsymbol{p}_{\text {rand }}$ is reachable. This technique allows for better spatial exploration of $\mathcal{W}$.

Among all nodes sampled in order to reach $\boldsymbol{p}_{\text {rand }}$ only the subset that guarantees the reachability of $\boldsymbol{p}_{\text {goal }}$ is considered. Then, the closest node $\boldsymbol{q}_{n e w}$, as defined by the euclidean norm, is kept to grow the tree (cf. Fig. 5b). Irrelevant tree growth is therefore avoided.

To introduce goal research bias, from each created node $\boldsymbol{q}_{\text {new }}$, a new set of inputs is applied to try and connect the tree with $\boldsymbol{p}_{\text {goal }}$. If the process is successful, a new node $\boldsymbol{q}_{\text {reach }}$ is added to the tree (cf. Fig. 5c). Else, a new point $\boldsymbol{p}_{\text {rand }}$ is drawn and the process starts again.

\section{ii) Varying Tissue Stiffness}

To account for the needle cutting angle and variable curvature, the modified unicycle model presented in section II-B 


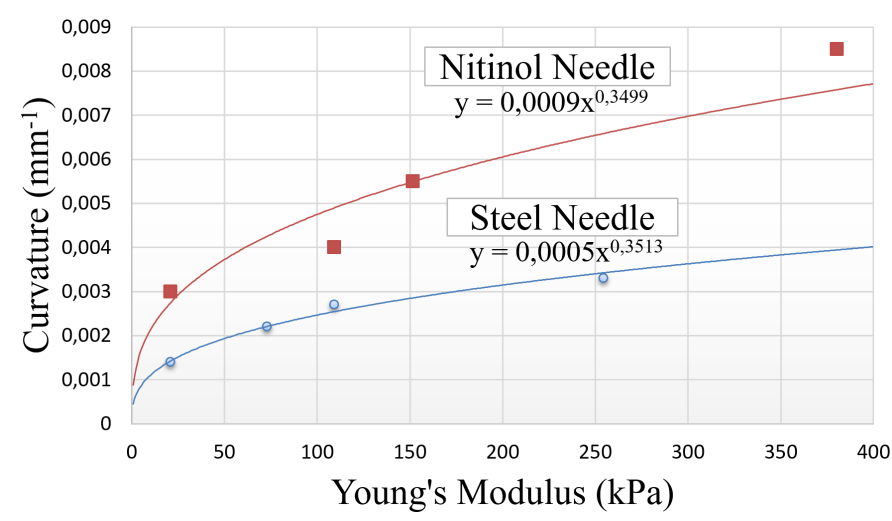

Fig. 6: 24 Gauge needle curvature as a function of the tissue stiffness.

is integrated for path generation. From pre-operative or intraoperative elastography measurements, a tissue stiffness map can be computed. This map enables the RRT algorithm to consider the expected curvature changes during the planning. Because the current curvature is estimated online by the observer detailed in section II-B, this value is preferred over elastography measurements. Therefore, the path planning is classically done with the estimated radius of curvature in $q_{\text {init }}$. However, when encountering a significant stiffness change (e.g. leading to a change in curvature $\geq 0.001 \mathrm{~mm}^{-1}$ ), the curvature used for path planning is computed from the stiffness-curvature relationship detailed in Fig. 6. Indeed, after a $4 \mathrm{~cm}$ deep insertion, a change in curvature of $0.001 \mathrm{~mm}^{-1}$ would roughly cause an unexpected $1 \mathrm{~mm}$ deflection (resp. $4 \mathrm{~mm}$ for an $8 \mathrm{~cm}$ insertion). The deflection at a certain depth for a pure insertion can be computed from equation (3) by considering an in plane deflection $\left(z_{\mathcal{F}_{t}}=0, y_{\mathcal{F}_{t}} \geq 0\right)$. Under the condition $x_{\mathcal{F}_{t}}<\frac{1}{\kappa}$, at the insertion depth $x_{\mathcal{F}_{t}}$, the deflection $y_{\mathcal{F}_{t}}$ is the smaller solution of $y_{\mathcal{F}_{t}}^{2}-\frac{2}{\kappa} y_{\mathcal{F}_{t}}+x_{\mathcal{F}_{t}}^{2}=0$.

If no drastic curvature change is expected, elastography measurements may prove unnecessary because of their lack of precision. In that case, the online curvature estimation may be sufficient and more accurate than SWE measurements.

The stiffness-curvature relationship illustrated in Fig. 6 has been obtained empirically. Five pure insertions have been done per homogeneous phantom of known elasticity (and per needle) and tracked using 3D ultrasound imaging. The radius of curvature is estimated online with the method developed in section II-B. The median value of this estimation is kept as the measurement of the curvature for a given phantom. The needle curvature varies with respect to the tissue stiffness, the needle flexibility, and the tip asymmetry [29].

In these first trials, the stiffness map is computed from preoperative SWE imaging. Although 3D SWE acquisitions can be acquired with a suitable probe, a first approach adopted here is to extrapolate 2D SWE acquisitions to obtain a locally estimated 3D stiffness map.

\section{iii) Choice of an Optimal Motion Plan}

After computing efficiently a set of successful needle tip trajectories via the RRT, a second in-depth analysis can be
TABLE I: General correlation* between objectives and cost function coefficients

\begin{tabular}{|l|c|c|c|c|}
\hline & $\begin{array}{c}\text { Targeting } \\
\text { Precision }\end{array}$ & $\begin{array}{c}\text { Obstacle } \\
\text { Avoidance }\end{array}$ & $\begin{array}{c}\text { Measurements } \\
\text { Quality }\end{array}$ & $\begin{array}{c}\text { Tissue Trauma } \\
\text { Minimization }\end{array}$ \\
\hline$c_{1}$ & ++ & - & - & - \\
\hline$c_{2}$ & - & ++ & none & - \\
\hline$c_{3}$ & - & none & ++ & - \\
\hline$c_{4}$ & - & - & - & ++ \\
\hline \multicolumn{4}{|c|}{++ strong positive correlation. } \\
$-:$ negative correlation.
\end{tabular}

* The correlation may change depending on the setting.

started. For each successful path $\Phi$, the multi-rate unscented Kalman filter is started from the state $\boldsymbol{q}_{\text {init }} \in \mathbb{R}^{7}$ with its estimate covariance matrix $\boldsymbol{P}_{k \mid 0} \in \mathbb{R}^{7 \times 7}$, with the control strategy computed for $\Phi$. No measurement is considered in that case, as the path is only simulated. Only a random subset of successful paths are recomputed this way with a fine precision inherited from the estimation algorithm presented in II-B.

The optimal motion plan must be chosen, among these computed paths, as a trade-off between the following correlated criteria:

- reach the target with precision;

- avoid obstacles;

- maximize the needle visibility in the US volumes;

- limit the tissue trauma.

This trade-off can be formalized as the research of the path $\Phi$ that minimizes the cost function:

$J(\Phi)=c_{1} d_{\text {target }}(\Phi)+c_{2} p_{\text {col }}(\Phi)+c_{3} \bar{R}(\Phi)+c_{4} c_{\text {command }}(\Phi)$,

where $c_{1}, c_{2}, c_{3}, c_{4} \in \mathbb{R}_{+}^{4}$ are coefficients tuned by the user to prioritize the objectives depending on the application. $d_{\text {target }}$ is the final euclidean distance to the target. $p_{c o l}(\Phi)$ detailed in (8) is the probability to collide with an obstacle. $\bar{R}(\Phi)$ detailed in (7) is inversely proportional to the needle visibility. $c_{\text {command }}(\Phi)$ detailed in (5) is the control cost.

For all the insertions presented in this paper, the coefficients $c_{i}$ were set so as to give equal importance to all objectives, i.e., normalize the variation range of the four terms composing $J(\Phi)$. However, the user can change them in real-time depending on the desired objectives. The correlation between these coefficients and the control objectives are detailed in Table I. These correlations are computed from path planning in a static environment with one obstacle, one target and one static needle. They represent the average variation of the objectives costs, when the value of a coefficient $c_{i}$ is increased. A visual illustration of this process is proposed in a video ${ }^{1}$.

\section{iv) Tissue Damage}

An easily overlooked issue for needle steering in biological tissue is the possible winding of the tissue around fine needles. This winding is caused by the rotation of the needle shaft inside the tissue. The needle tip may suffer from the accumulation of fibers that cover its bevel and prevents the robot from controlling the needle. Another effect of such winding

\footnotetext{
${ }^{1}$ Available at https://youtu.be/aI5-jT2cbRY.
} 
is a strong adherence of the needle shaft to its surroundings. This may result in increased tissue trauma, unexpected needle behavior or even the inability to further insert or retract the needle [22].

Therefore, in this paper, we wish to compute smooth paths, that reduce the control cost $c_{\text {command }}(\Phi)$ [rad] defined as the amount of rotation in the tissue.

$$
c_{\text {command }}(\Phi)=\sum_{i}\left(\left|u\left(\Phi_{i}\right)\right| \cdot T\left(\Phi_{i}\right)\right),
$$

where the trajectory $\Phi$ is composed of subinsertions $\Phi_{i}$ of constant rotation speed $u\left(\Phi_{i}\right)[\mathrm{rad} / \mathrm{s}]$ over a period $T\left(\Phi_{i}\right)$ [s].

\section{v) Measurement Uncertainties}

The needle visibility is a critical criterion when using 3D US imaging. The visibility of the needle in 3D B-mode ultrasound imaging depends on factors such as the distance between the needle and the probe, the incidence angle of the US waves on the needle and the shadowing artifacts. Although a precise confidence map can be computed to take into account shadowing or luminous artifacts [11], we consider in a first approach that the image quality decreases linearly with the observation depth.

The quality is proportional to the $\mathrm{x}, \mathrm{y}, \mathrm{z}$ terms of the measurement noise covariance matrix $\boldsymbol{R}$ of the Kalman filter detailed in section II-B. As in [19], the standard deviations of the tip cartesian coordinates measurements are chosen equal to

$$
\sigma_{\text {actual }}=\left(1+a \frac{d}{d_{\max }}\right) \sigma_{\text {optimal }},
$$

where $\sigma_{\text {optimal }}$ is the standard deviation of the Cartesian coordinates in the best case (close to the transducer). $\sigma_{\text {actual }}$ is its actual value at the given tip position. $d$ is the distance of the given tip position to the transducer. $d_{\max }$ is the acquisition depth of the transducer ( $8 \mathrm{~cm}$ here). $a \in \mathbb{R}^{+}$reflects the quality loss of the signal, empirically set to 1 .

The needle visibility along a path $\Phi$ is characterized by the mean measurements uncertainty

$$
\bar{R}(\Phi)=\operatorname{mean}_{\Phi}\left(\sigma_{\text {actual }}\right) .
$$

\section{vi) Collision Probability}

As the needle is inserted, its future behavior is uncertain. Not only because its current state is only estimated, but also because the model cannot fully represent the real system behavior. This may lead to unexpected obstacle collision in spite of an initially adequate planning. If a simple geometric obstacle clearance can be introduced, it does not take into account the physical behavior of the needle and the probability of collision. This is why obstacle avoidance is handled as follows.

The tip pose estimation provided by the Kalman filter described in section II-B is used to propagate the state uncertainties.

The multi-rate unscented Kalman filter is started from the state $\boldsymbol{q}_{\text {init }} \in \mathbb{R}^{7}$ with its estimate covariance matrix $\boldsymbol{P}_{k \mid 0} \in \mathbb{R}^{7 \times 7}$, with the control strategy computed for $\Phi$. No measurement is considered in that case, as the path is

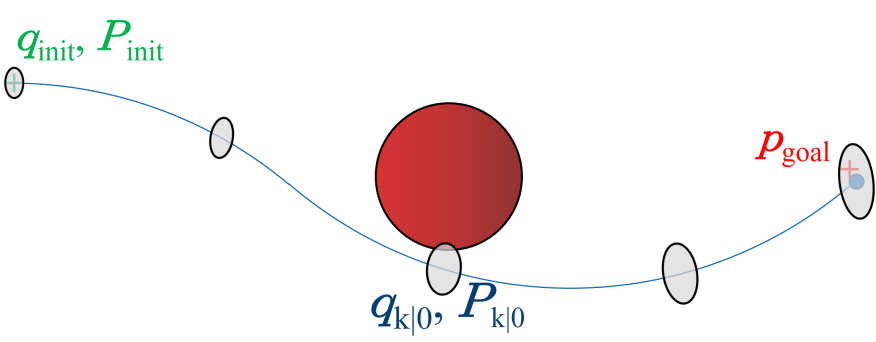

Fig. 7: Uncertainties propagation along a mean path computed by the RRT.

only simulated. The estimate covariance matrix $\boldsymbol{P}_{k \mid 0}$ is thus computed along $\Phi$, and expresses the possible divergence of the real path around $\Phi$ (see Fig. 7).

The collision probability of a planned path $\Phi$ with an obstacle $p_{c o l}$ is computed from the heuristic:

$$
p_{\text {col }}(\Phi)=\max _{k}\left(\int_{\text {obstacle }} \mathcal{N}\left(\boldsymbol{\mu}_{\boldsymbol{k}}(\boldsymbol{\Phi}), \boldsymbol{\Sigma}_{\boldsymbol{k}}(\boldsymbol{\Phi})\right)\right),
$$

where $\boldsymbol{\mu}_{\boldsymbol{k}} \in \mathbb{R}^{3}$ is the mean estimated cartesian position of the needle tip after $k$ iterations. $\boldsymbol{\Sigma}_{\boldsymbol{k}} \in \mathbb{R}^{3 \times 3}$ is the estimation covariance matrix of the needle tip cartesian coordinates. $\boldsymbol{\Sigma}_{\boldsymbol{k}}$ being the submatrix of $\boldsymbol{P}_{k \mid 0}$ such that

$$
\boldsymbol{P}_{k \mid 0}=\left(\begin{array}{c|c}
\boldsymbol{\Sigma}_{\boldsymbol{k}} & \times \\
\hline \times & \times
\end{array}\right)
$$

This collision probability has a physical meaning that allows a user to set precise goals (e.g. realize an insertion with a collision probability smaller than $5 \%$ ).

\section{vii) Fast Replanning}

As discussed in the aforementioned section, the needle behavior is prone to uncertainties. Besides, the environment itself is likely to change unexpectedly (e.g. tissue deformation, target or obstacle movement, etc.). In this context, pre-operative planning seems unsuitable for robust, intra-operative needle steering.

We propose to take a fast replanning approach centered around the current pose estimation provided at $100 \mathrm{~Hz}$ by the multi-rate unscented Kalman filter $\hat{\boldsymbol{q}}_{k \mid k}$ (i.e. $\boldsymbol{q}_{i n i t}=\hat{\boldsymbol{q}}_{k \mid k}$ ). Such an approach gives to the planning algorithm the ability to adapt to unexpected changes in the system through computation of new paths. Besides, the path planning considers the new trust we have in the current tip pose estimate. If the estimate covariance matrix $P_{\text {init }}$ of $q_{\text {init }}$ increases following missing or incorrect measurements, the obstacle collision computation is updated accordingly. The obstacle is then avoided with a greater margin.

\section{EXPERIMENTAL ASSESSMENT}

62 insertions with a 24 Gauge nitinol beveled-tip needle were made in various media to validate the robustness of the proposed solution for fine superelastic needles. 5 insertions were made with a 18 Gauge steel biopsy needle to evaluate the approach when using stiffer needles. 


\section{A. Hardware and Software Implementation}

The needle is inserted at the constant speed of $0.5 \mathrm{~mm} . \mathrm{s}^{-1}$ by the Prosper robot developed by N. Hungr [23] for prostate brachytherapy. The rotation inputs, computed by the iteration bounded RRT in the interval $[-\pi / 3, \pi / 3] \mathrm{rad}^{-1}{ }^{-1}$, are directly used in the steering algorithm. This is made possible by the fast replanning approach which recomputes the best inputs for the current system configuration (at a frequency $\geq 1 \mathrm{~Hz}$ and provided that the insertion speed remains low).

The same modified unicycle model is used for observation and path planning. For all insertions, the cutting angle is set equal to its average value of $3^{\circ}$, as deduced from the data fitting done in [19]. In this experimental context, the torsion of the needle around its axis, as it is rotated in the tissue, is considered negligible. Indeed, the needle is constantly inserted as it rotates, which helps release the torsional energy it may accumulate [30].

Throughout the 67 insertions performed, the segmentation algorithm weights were set as $w_{p_{1}}=3$ and $w_{p_{2}}=1$. The ROI tube diameter was chosen equal to $2.4 \mathrm{~mm}$ in all reported experiments.

The 24 Gauge nitinol needles have a $30^{\circ}$ beveled-tip. These needles are treated to be more echogenic. Their surface is either coated with polyurethane foam as in [19] or etched with a laser as described in [13]. The 18 Gauge steel needles also have a $30^{\circ}$ beveled-tip, but they do not have any surface treatment. The ultrasound volumes are acquired at $1 \mathrm{~Hz}$ with a 3D end-fire probe 4DEC-9/10 used with the Ultrasonix Sonix RP ultrasound system. The US volume voxels are cubes with $0.4 \mathrm{~mm}$ edges. The pre-operative SWE measurements are acquired through the SuperLinear ${ }^{\mathrm{TM}}$ SLH20-6 probe used with the SuperSonic Imagine Aixplorer ${ }^{\circledR}$ ultrasound system.

The phantoms are made of gelatin, agar or ex-vivo tissue samples enclosed in gelatin. Heterogeneous agar phantoms have also been made, which consist in two homogeneous halves fused together on about half a millimeter. The targets are, either virtual static points set in the ultrasound volume, or aluminum balls of radius $1 \mathrm{~mm}$ implanted in the tissue to allow for contact detection.

To simulate a scenario in which the needle might not be initially perfectly oriented or where the target moves, the targets are misaligned with the initial needle orientation. Target misalignment is defined as the distance of the target to the line that extrapolates the initial needle pose (see the figure in Table II).

The proposed solution has been developed in $\mathrm{C}++$ in the CamiTK framework (see [31] for more details). All blocks illustrated in Fig. 1 are designed to run asynchronously in independent threads. To achieve the desired performance, we used the CPU multiprocessing library OpenMP, with NVIDIA $^{\circledR}$ GPU parallel computing platform CUDA ${ }^{\circledR}$. The code runs on a laptop with an Intel ${ }^{\circledR}$ Core $^{\mathrm{TM}}$ I5-8300H CPU with an NVIDIA ${ }^{\circledR}$ GeForce $^{\circledR}$ GTX 1050 Ti GPU. The needle tip pose and curvature observer runs at $100 \mathrm{~Hz}$, the needle tip segmentation is done every acquired frame and the path replanning is done at about $1 \mathrm{~Hz}$.

\section{B. Experimental Protocol}

In the experimental protocol, the user sets up the US imaging system, then inserts the needle a few millimeters into the tissue. The needle must be manually localized in the 3D US volume as two points, one being the tip. The user can then provide the theoretical needle curvature, or the needle type and a stiffness map. The target (mandatory) and obstacles (optional) can be added as spheres of a chosen radius. The RRT may then be started and the possible paths visualized. The four coefficients of the cost function can be tuned in real-time to prioritize objectives. On the user command, the robotized insertion begins, following the RRT results until the target is attained or becomes unreachable.

The initial static target misalignment was voluntarily chosen significantly high in comparison with the maximum deflection achievable in each phantom. This proves the ability of the algorithm to steer the needle in difficult conditions. We believe this validates the capacity to also attain moving targets that are initially less misaligned.

\section{Insertion Setting}

\section{i) Homogeneous Phantom and nitinol Needle}

A first validation is made in homogeneous gelatin or agar phantoms to validate the proposed solution in a simple configuration. In this medium, the needle visibility is at its highest. No fibers or tissue heterogeneity are expected and the tissue elasticity and estimated needle curvature are well known.

For a part of the experiments (without obstacle), the target is a physical object. Here, it is an aluminum ball of $1 \mathrm{~mm}$ radius (i.e. matching the $2 \mathrm{~mm}$ spatial resolution of the probe used here), soldered to a wire. When the needle touches the target, a circuit is closed and a buzzer rings, certifying a good mechanical contact with the target. This method proves to be more reliable and precise than manual visual segmentation of the needle tip when the targeting precision is submillimetric.

20 insertions were made in a $15 \%$ gelatin phantom $(E \sim$ $150 \mathrm{kPa}), 10$ with an obstacle. The theoretical needle curvature is $\kappa=0.0055 \mathrm{~mm}^{-1}$ (see Fig 6). This corresponds to a maximum final deflection of $19 \mathrm{~mm}$ for a $8 \mathrm{~cm}$ insertion without rotation. The high curvature allows for large deflections and complex helical needle steering with significant obstacle avoidance. The aim of these experiments is not to match a given tissue stiffness, but rather to bring a proof of concept of our algorithm functioning.

\section{ii) Multi-Layered Phantom and nitinol Needle}

A second set of insertions made into a multi-layered phantom aims to validate the robustness of the proposed method to drastic tissue stiffness changes.

No obstacle is defined in the phantom. A stiffness map is created by the user after estimating the stiffness of the phantom layers with pre-operative SWE imaging (see Fig. 9b).

10 insertions were made in an agar phantom with 5\% $(E \sim$ $380 \mathrm{kPa})$ and $2.5 \%(E \sim 110 \mathrm{kPa})$ agar layers (see Fig. 9) for theoretical needle curvatures of $\kappa=0.0080 \mathrm{~mm}^{-1}$ and $\kappa=0.0050 \mathrm{~mm}^{-1}$ respectively (see Fig 6). In 5 of them, the 
stiffness map is taken into account in the planning. In 5 others, the curvature is only initialized as $\kappa=0.0080 \mathrm{~mm}^{-1}$.

The phantom stiffness is chosen high to better evaluate the impact of a variation of tissue stiffness on the steering ability in the perspective of a real clinical application. For instance, in prostate brachytherapy, the modification of stiffness between the perineum $(\sim 30 \mathrm{kPa})$ and a cancerous prostate $(\sim 100 \mathrm{kPa})$ yields a similar evolution in needle curvature which nearly doubles.

iii) Ex-Vivo Tissue Sample, Soft Phantom and nitinol Needle A third set of insertions made into pork tenderloin aims to evaluate the performance of the proposed method with real tissue. No obstacle was defined in the tissue. The tissue structure is fibrous. The medium is therefore very echogenic which greatly decreases the needle visibility in the US volumes. Besides, the needle behavior is more uncertain than in homogeneous phantoms.

25 insertions were made in pork tenderloin $(E \sim 25$ $\mathrm{kPa}$ ) encased in gelatin. The theoretical needle curvature is $\kappa=0.0025 \mathrm{~mm}^{-1}$ (see Fig 6), corresponding to a final deflection of $8 \mathrm{~mm}$ for a $8 \mathrm{~cm}$ insertion without rotation. For a fair comparison with homogeneous phantom insertions, 7 additional insertions were made into a soft $7 \%$ gelatin phantom $(E \sim 30 \mathrm{kPa})$ with similar theoretical curvature and comparable target misalignment.

\section{iv) Stiff needles}

The validation for stiff needles is made in homogeneous agar phantom to validate the proposed solution in a simple configuration. As the needle did not benefit from surface treatment, its visibility is highly affected by artifacts in the US volume.

The target is a virtual object. No obstacle is defined in the phantom as the curvature of the needle is very low.

5 insertions were made in a $2.5 \%$ agar phantom $(E \sim 110$ $\mathrm{kPa})$. The theoretical needle curvature is $\kappa=0.001 \mathrm{~mm}^{-1}$. This corresponds to a maximum final deflection of $3 \mathrm{~mm}$ for a $8 \mathrm{~cm}$ insertion without rotation.

\section{Results}

Detailed experimental results can be found in Table II, while Fig. 8 and a video ${ }^{2}$ illustrate real insertion cases. The target is either virtual and defined as a point in space by the user, or physical and embedded in the tissue. The distance to the target is computed as the euclidean distance between the manually segmented final tip position and the target. The manual segmentation for one needle configuration is done with a standard deviation of $0.3 \mathrm{~mm}$. Therefore, the results in this paper must be appreciated in the light of this manual segmentation repeatability.

\section{i) Homogeneous Phantom and nitinol Needle}

Without obstacle, all insertions were successful (i.e. reached the physical target) despite the large target misalignment. This demonstrates a submillimeter targeting accuracy. With obstacle

\footnotetext{
${ }^{2}$ Available at https://youtu.be/RtvYRDtAb70.
}

TABLE II: Needle Steering Results

\begin{tabular}{|c|c|c|c|}
\hline Insertion Setting & $\begin{array}{c}\begin{array}{c}\text { Number } \\
\text { of insertions }\end{array} \\
\text { [mean insertion length }(\mathrm{mm})]\end{array}$ & $\begin{array}{c}\begin{array}{c}\text { Initial target } \\
\text { misalignment (mm) }\end{array} \\
\\
\end{array}$ & $\begin{array}{c}\text { Targeting } \\
\text { error }(\mathbf{m m})\end{array}$ \\
\hline i 1 Homogeneous gelatin & $\begin{array}{c}10 \\
{[84]}\end{array}$ & $\begin{array}{l}11.8 \pm 3.0 \\
{[5.5: 16.3]}\end{array}$ & $<1^{*}$ \\
\hline 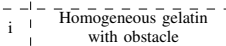 & $\begin{array}{l}-104] \\
{[93]}\end{array}$ & $\begin{array}{r}-5.5 \pm 4.2 \\
{[0.9 ; 12.6]}\end{array}$ & $\begin{array}{c}-\overline{1.3 \pm} 0 . \overline{6}^{* * *} \\
{[0.5 ; 2.0]}\end{array}$ \\
\hline $\begin{array}{lll}\text { ii } & \text { Bi-layered agar }\end{array}$ & $\begin{array}{c}5 \\
90]\end{array}$ & $16.0 \pm 10.0$ & $3.0 \pm 1.5^{* * *}$ \\
\hline 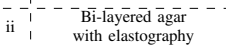 & {$[93]$} & $\begin{array}{l}-1 \overline{2} \pm 4.6^{-} \\
{[10.3 ; 21.0]}\end{array}$ & $\begin{array}{c}1.5 \pm 0.9^{* *} \\
{[0.3 ; 2.9]}\end{array}$ \\
\hline iii Homogeneous soft gelatin & $\begin{array}{c}7 \\
{[87]}\end{array}$ & $\begin{array}{l}6.3 \pm 2.4 \\
{[2.8 ; 8.9]}\end{array}$ & $<1^{*}$ \\
\hline \begin{tabular}{l|l} 
& Pork Tenderloin \\
iii
\end{tabular} & $\begin{array}{l}25 \\
{[79]}\end{array}$ & $\begin{array}{l}4.6 \pm 1.8 \\
{[1.1 ; 7.5]}\end{array}$ & 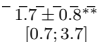 \\
\hline
\end{tabular}

Needle steering results for a 24 Gauge beveled-tip nitinol needle.

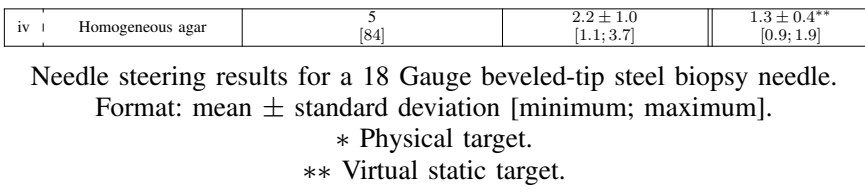

avoidance, the targeting error increased to $1.3 \pm 0.6 \mathrm{~mm}$, which is expected, as both objectives are correlated (see Table I). Examples of insertions can be found in Fig. 8a and 8b.

\section{ii) Multi-Layered Phantom and nitinol Needle}

In multi-layered phantoms, the average targeting error decreases to $3.0 \pm 1.5 \mathrm{~mm}$ as the tissue heterogeneity induces significant curvature changes. Introducing the elastography measurements induces a twofold decrease of the targeting error (to $1.5 \pm 0.9 \mathrm{~mm}$ ) by limiting curvature misestimation. An example of insertion can be found in Fig. 8c.

\section{iii) Ex-Vivo Tissue Sample and Soft Phantom and nitinol} Needle

In soft homogeneous phantoms, a submillimeter targeting accuracy can be retrieved. However, when considering insertions made into similar stiffness pork tenderloin tissue, the targeting error increases to $1.7 \pm 0.8 \mathrm{~mm}$. This is mainly due to the tissue fibers and fat layers which disrupt the needle steering by provoking small local buckling and unmodeled structural interactions. An example of insertion in pork can be found in Fig. 8d.

\section{iv) Stiff Needles}

For stiff needles, the steering response is greatly diminished. The initial target displacement has therefore been set to a few millimeters. In soft homogeneous phantoms without obstacle, the targeting performance is, as expected, lower than that of nitinol needles, with a targeting error of $1.3 \pm 0.4 \mathrm{~mm}$.

\section{Discussion}

The average targeting precision is promising in regard to similar work [13] with a twofold increase in precision in exvivo biological tissue, and an overall steadier performance to medium variation. Further result-based comparison with other works might be irrelevant as the experimental conditions for needle steering vary greatly. 


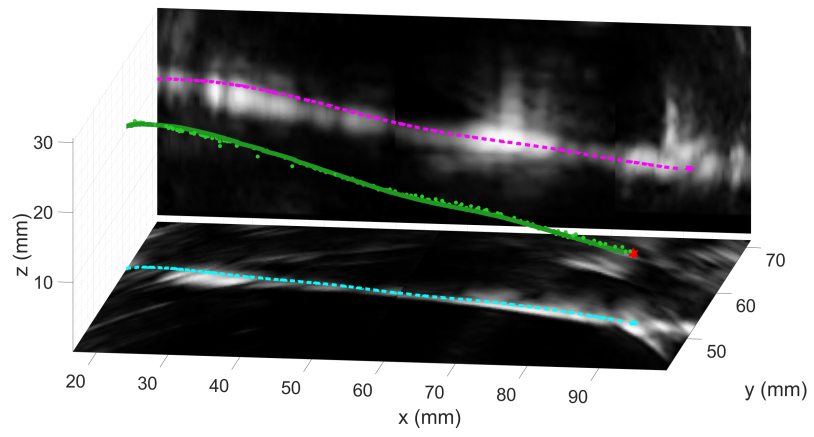

(a) Insertion in homogeneous gelatin phantom.

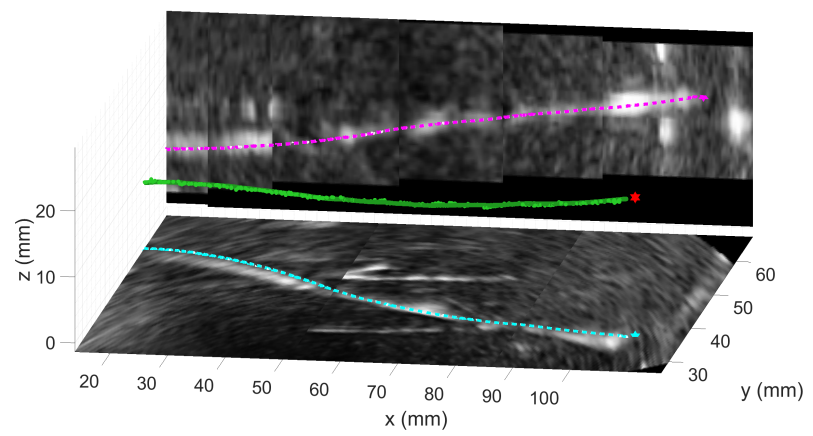

(c) Insertion in heterogeneous agar phantom with elastography.

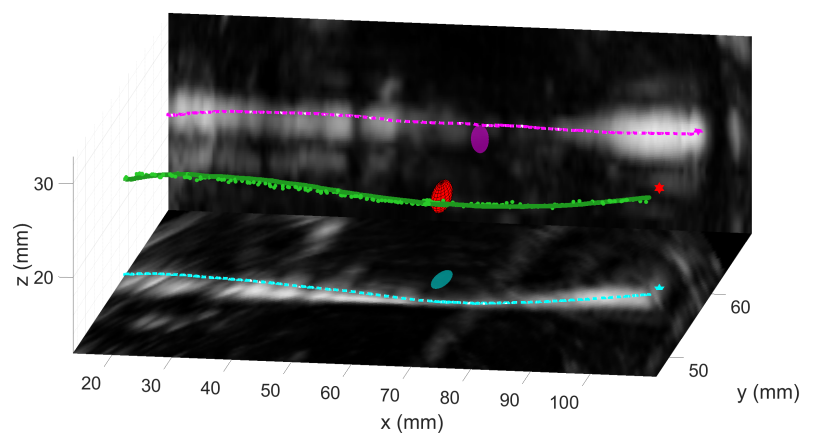

(b) Insertion in homogeneous gelatin phantom with obstacle (red sphere).

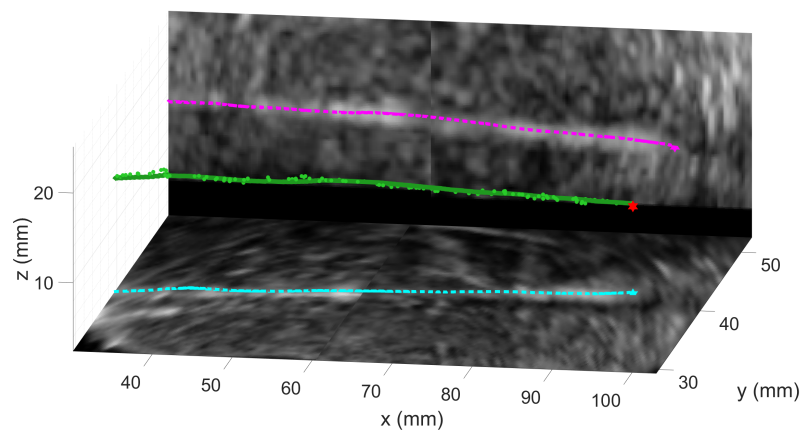

(d) Insertion in ex-vivo tissue.

\begin{tabular}{|ll|ll|}
\hline- & Estimated needle tip trajectory (3D) & $\bullet$ & Needle tip segmentation \\
----- & Estimated needle tip trajectory (Projected XY) & $*$ & Target \\
---- & Estimated needle tip trajectory (Projected XZ) & & Obstacle (optional) \\
\hline
\end{tabular}

Fig. 8: 3D representation of needle steering in various media. For better visualization, the $2 \mathrm{D}$ ultrasound pictures represented here are a combination of $2 \mathrm{D}$ slices of a $3 \mathrm{D}$ volume, acquired at the end of the needle insertion.

\section{A. Methods}

In the following subsections, we will discuss the methods in the light of the experimental assessment.

1) Needle Tip Localization: In all insertions, the needle tip localization proved to be robust to low visibility. This can be seen especially for trials in ex-vivo tissue and with steel biopsy needles which suffer from significantly high intensity artifacts (see Fig. 10).

Partial needle shaft loss and lack of contrast are compensated by the Bayesian classification. It adapts the tip classification accordingly to the ROI histogram. The same observation can be made when the follow the leader hypothesis is incorrect and only a part of the needle shaft is in the ROI.

The good performance of the tip localization can also be explained by the intrinsic robustness of each software block and their interaction (see Fig. 1). Indeed, when the needle tip is not visible and the segmentation fails, the other algorithms rely on the remaining data. In that case, the curvature cannot be estimated online and is chosen as constant over time. When the tip becomes visible again, the segmentation recovers the tip in the ROI updated by the observer, and provides feedback to the other processes.
2) Path Planning: The adaptative path planning enables obstacle avoidance with little impact on the targeting accuracy and adapts itself to heterogeneities and uncertainties. These considerations allow for a robust needle steering motion planning specifically adapted for insertions in biological tissue. Indeed, in such tissue the needle may encounter fibers and fat layers that provoke discontinuities in the needle behavior.

The uncertainties are taken into account through two mechanisms. First, the loop formed by the needle tip pose estimation and the path planning enables the consideration of the tip estimation covariance. Second, the fast replanning approach takes into account the updated state of the needle and that of its environment.

The algorithm is robust to tissue stiffness changes as the heterogeneities are considered through both curvature estimation and prediction. This leads to the computation of paths with depth-dependent curvature. This SWE-based motion plan is introduced for the first time in the context of needle steering. Even if the stiffness map itself may not be accurate, its information is valuable. It helps to estimate the reachable space (see Fig. 9a) and increases the precision of the steering.

One important limitation of fine needle steering in biological tissue is the winding of fibers around the needle 


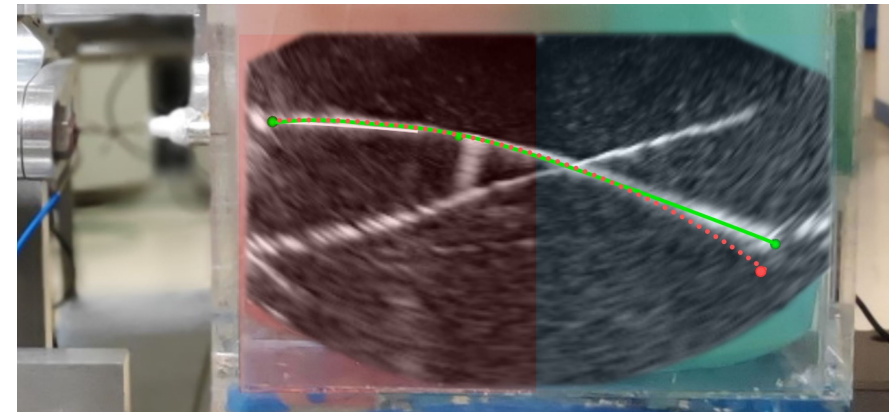

(a) Steering of a nitinol needle into a heterogeneous agar phantom with superimposed US volume. Without elasticity map, the real trajectory (green curve) differs from the expected trajectory (dotted red curve) and misses the target (red sphere). With elasticity map, the unattainability of the target is detected before insertion.

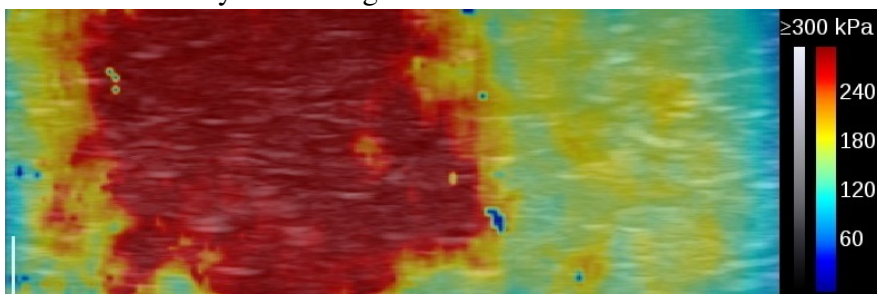

(b) Corresponding sample of shear wave elastography imaging.

Fig. 9: Illustration of the usefulness of the elasticity map in a heterogeneous medium. The target is placed out of the true reachable space. The red part of the phantom is stiffer than the blue one. A trace from a previous insertion can be seen, the algorithm remains robust to its presence.

tip, making duty cycling approaches very challenging. To limit such winding, the proposed solution guides the needle following smooth helical paths that limit the needle rotation. However, due to the stochastic exploration of the RRT, small oscillations of the rotation angle occur when the needle is being inserted. Therefore, better continuity of the needle path could be introduced to further reduce unnecessary changes in the motion plan.

\section{B. Clinical applicability}

This work is focused on using 3D US imaging that we believe is a step forward from 2.5D imaging in regard to clinical applicability. However, in a clinical setup, the position of the probe may differ from what is presented in this paper and could negatively affect the needle tip localization. In real applications, this potential issue should be limited by the practitioner efforts to place the probe to ensure good needle visibility, as is naturally often done in regular needle procedures.

Insertions with superelastic needles rods have been presented in this paper to achieve greater deflection than clinically available needles. In real tissue, the steering of steel needles is reduced to a few millimeters and limits tissue motion compensation and obstacle avoidance. Despite this, the utility of the algorithm still remains as the path is recomputed to account for the obstacle and target motion, while minimizing tissue damage and possible buckling.
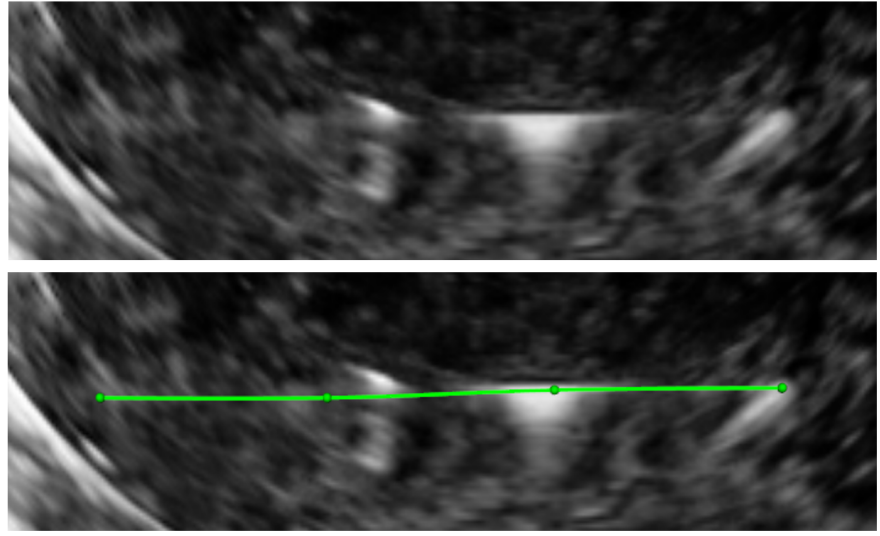

Fig. 10: Example of bad visibility of a steel needle in a 3D ultrasound volume. Top picture: original volume. Bottom picture: automatically needle tip tracking in green.

To fulfill more constrained objectives, the steering response could be amplified. First, the experiments would benefit from a greater depth of insertion, constrained by the clinical application. Also, clinically compatible needle design and actuation strategies can be selected to raise the needle curvature value [29]. Finally, the steering capability is increased for clinical insertions in stiff tissue (e.g. the prostate gland).

The use of SWE could also be translated to a clinical scenario (e.g. prostate brachytherapy). A 3D map of the tissue stiffness could be acquired preoperatively or intraoperatively while the insertion is stopped. During the insertion, tissue tracking (e.g. prostate tracking [32]) could then be used to update the elasticity map to the tissue deformation.

A possible clinical application of the proposed method is the avoidance of the pubic arch in the case of prostate brachytherapy. This could be done to reach previously unattainable regions of the prostate while minimizing tissue trauma, and accounting for the stiffness difference between the prostate and the surrounding tissue.

In this proof of concept project, the insertion speed is set equal to $0.5 \mathrm{~mm} . \mathrm{s}^{-1}$. It may seem impractical for long insertions. However, simulations carried out at $1.5 \mathrm{~mm} . \mathrm{s}^{-1}$ validate the performances at a higher insertion speed. That is, under the condition that the tissue dynamics does not significantly affect the insertion at the chosen velocity.

\section{CONCLUSION}

The proposed solution is a promising step towards robust needle steering into soft and heterogeneous tissue. The interconnection between estimation, segmentation, path planning and asynchronous measurements increases the overall system robustness.

It allows for needle steering with poor needle visibility and temporary needle loss, through state estimation, adaptative needle segmentation and measurement quality mapping. This is necessary when working with 3D US imaging systems which have low sampling rate, low spatial resolution and are subject to noise and artifacts. 
The proposed solution accounts for uncertainties in the needle-tissue interaction through curvature estimation, patientspecific elasticity mapping, state covariance estimation, collision probability computation and fast path replanning.

The chosen steering approach may also cause less trauma to the tissue, by preferring helical paths over a duty cycling approach. Four control parameters can be tuned in real-time by the user to easily prioritize objectives. In practice, such tuning may depend on the intervention or on the stage of the intervention.

Future work will involve improving the proposed solution by incorporating clinical needs such as considering patient breathing, tissue deformation and putting the surgeon in the loop. Trials on anatomic subjects will be carried out to further demonstrate the clinical applicability of this work.

\section{REFERENCES}

[1] C. Rossa and M. Tavakoli, "Issues in closed-loop needle steering," Control Engineering Practice, vol. 62, pp. 55-69, May 2017.

[2] R. Alterovitz, T. Simeon, and K. Goldberg, "The Stochastic Motion Roadmap: A Sampling Framework for Planning with Markov Motion Uncertainty," in Robotics: Science and Systems III. Robotics: Science and Systems Foundation, Jun. 2007.

[3] E. Lobaton et al., "Planning curvature-constrained paths to multiple goals using circle sampling," in 2011 IEEE International Conference on Robotics and Automation. Shanghai, China: IEEE, May 2011, pp. 1463-1469.

[4] S. Patil, J. van den Berg, and R. Alterovitz, "Estimating probability of collision for safe motion planning under Gaussian motion and sensing uncertainty," in 2012 IEEE International Conference on Robotics and Automation. St Paul, MN, USA: IEEE, May 2012, pp. 3238-3244.

[5] W. Sun, S. Patil, and R. Alterovitz, "High-Frequency Replanning Under Uncertainty Using Parallel Sampling-Based Motion Planning," IEEE Transactions on Robotics, vol. 31, no. 1, pp. 104-116, Feb. 2015.

[6] J. Huang et al., "Imaging Artifacts of Medical Instruments in Ultrasound-Guided Interventions," Journal of Ultrasound in Medicine, vol. 26, no. 10, pp. 1303-1322, Oct. 2007.

[7] M. Abayazid et al., "Three-Dimensional Needle Steering Using Automated Breast Volume Scanner (ABVS)," Journal of Medical Robotics Research, vol. 01, no. 01, p. 1640005, Mar. 2016.

[8] J. Carriere et al., "Event-Triggered 3D Needle Control Using a ReducedOrder Computationally Efficient Bicycle Model in a Constrained Optimization Framework," Journal of Medical Robotics Research, vol. 04 no. 01, p. 1842004, Mar. 2019.

[9] T. K. Adebar, A. E. Fletcher, and A. M. Okamura, "3-D UltrasoundGuided Robotic Needle Steering in Biological Tissue," IEEE Transactions on Biomedical Engineering, vol. 61, no. 12, pp. 2899-2910, Dec. 2014.

[10] P. Mignon, P. Poignet, and J. Troccaz, "Using rotation for steerable needle detection in 3D color-Doppler ultrasound images," in 2015 37th Annual International Conference of the IEEE Engineering in Medicine and Biology Society (EMBC). Milan: IEEE, Aug. 2015, pp. 1544-1547.

[11] P. Chatelain, A. Krupa, and N. Navab, "Confidence-driven control of an ultrasound probe: Target-specific acoustic window optimization," in 2016 IEEE International Conference on Robotics and Automation (ICRA). Stockholm: IEEE, May 2016, pp. 3441-3446.

[12] J. Chevrie, A. Krupa, and M. Babel, "Real-time Teleoperation of Flexible Beveled-tip Needle Insertion using Haptic Force Feedback and 3D Ultrasound Guidance," in 2019 International Conference on Robotics and Automation (ICRA). Montreal, QC, Canada: IEEE, May 2019, pp. 2700-2706.

[13] P. Mignon, P. Poignet, and J. Troccaz, "Automatic Robotic Steering of Flexible Needles from 3D Ultrasound Images in Phantoms and Ex Vivo Biological Tissue," Annals of Biomedical Engineering, vol. 46, no. 9, pp. 1385-1396, Sep. 2018.

[14] M. Bernardes et al., "Robot-assisted automatic insertion of steerable needles with closed-loop imaging feedback and intraoperative trajectory replanning," Mechatronics, vol. 23, no. 6, pp. 630-645, Sep. 2013.
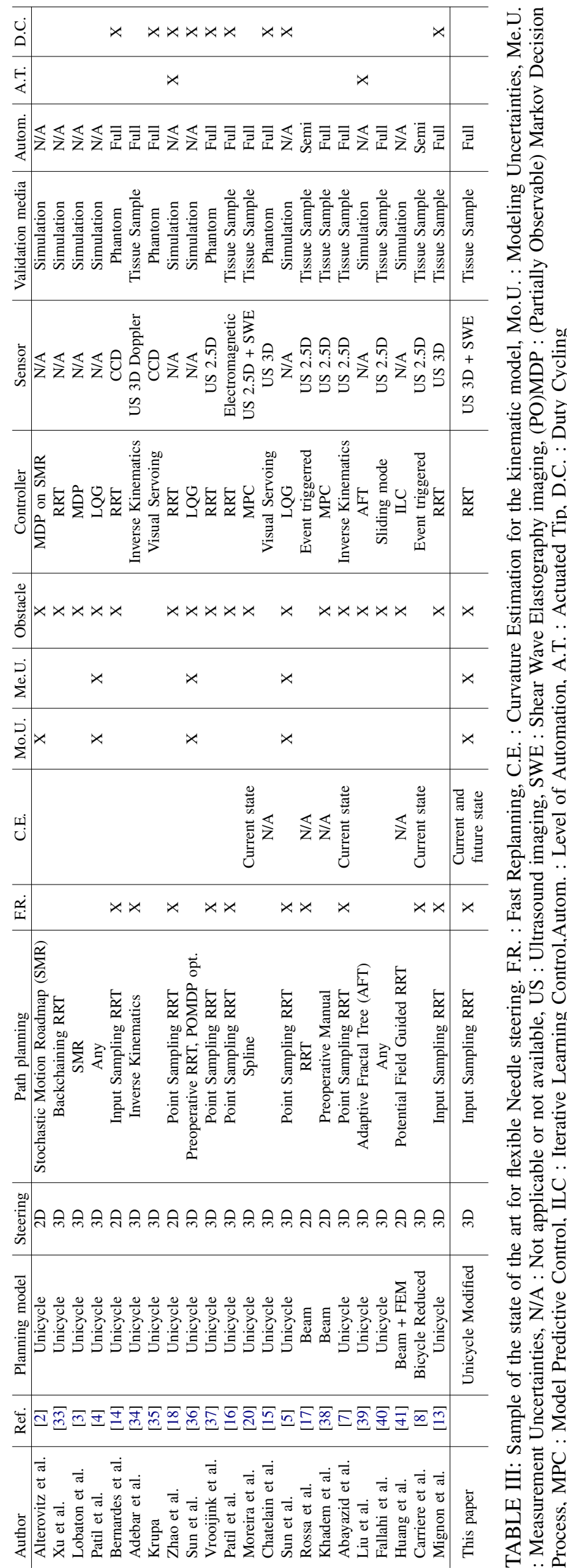
[15] P. Chatelain, A. Krupa, and N. Navab, "3D ultrasound-guided robotic steering of a flexible needle via visual servoing," in 2015 IEEE International Conference on Robotics and Automation (ICRA). Seattle, WA, USA: IEEE, May 2015, pp. 2250-2255.

[16] S. Patil et al., "Needle Steering in 3-D Via Rapid Replanning," IEEE Transactions on Robotics, vol. 30, no. 4, pp. 853-864, Aug. 2014.

[17] C. Rossa et al., "Constrained optimal control of needle deflection for semi-manual steering," in 2016 IEEE International Conference on Advanced Intelligent Mechatronics (AIM). Banff, AB, Canada: IEEE, Jul. 2016, pp. 1198-1203.

[18] Yan-Jiang Zhao et al., "Path planning for robot-assisted active flexible needle using improved Rapidly-Exploring Random trees," in 2014 36th Annual International Conference of the IEEE Engineering in Medicine and Biology Society. Chicago, IL: IEEE, Aug. 2014, pp. 380-383.

[19] G. Lapouge, J. Troccaz, and P. Poignet, "Multi-rate unscented Kalman filtering for pose and curvature estimation in 3D ultrasound-guided needle steering," Control Engineering Practice, vol. 80, pp. 116-124, Nov. 2018.

[20] P. Moreira and S. Misra, "Biomechanics-Based Curvature Estimation for Ultrasound-guided Flexible Needle Steering in Biological Tissues," Annals of Biomedical Engineering, vol. 43, no. 8, pp. 1716-1726, Aug. 2015.

[21] J. Engh et al., "Toward Effective Needle Steering in Brain Tissue," in 2006 International Conference of the IEEE Engineering in Medicine and Biology Society. New York, NY: IEEE, Aug. 2006, pp. 559-562.

[22] R. Tsumura et al., "Histological evaluation of tissue damage caused by rotational needle insertion," in 2016 38th Annual International Conference of the IEEE Engineering in Medicine and Biology Society $(E M B C)$. Orlando, FL, USA: IEEE, Aug. 2016, pp. 5120-5123.

[23] N. Hungr et al., "A 3-D Ultrasound Robotic Prostate Brachytherapy System With Prostate Motion Tracking," IEEE Transactions on Robotics, vol. 28 , no. 6 , pp. 1382-1397, Dec. 2012.

[24] M. Abayazid et al., "Integrating Deflection Models and Image Feedback for Real-Time Flexible Needle Steering," IEEE Transactions on Robotics, vol. 29, no. 2, pp. 542-553, Apr. 2013.

[25] R. J. Webster et al., "Nonholonomic Modeling of Needle Steering," The International Journal of Robotics Research, vol. 25, no. 5-6, pp. 509525, May 2006

[26] H. Younes, S. Voros, and J. Troccaz, "Automatic needle localization in 3D ultrasound images for brachytherapy," in 2018 IEEE 15th International Symposium on Biomedical Imaging (ISBI 2018). Washington, DC: IEEE, Apr. 2018, pp. 1203-1207.

[27] G. Lapouge et al., "Needle Segmentation in 3D Ultrasound Volumes Based on Machine Learning for Needle Steering," in The Hamlyn Symposium on Medical Robotics. The Hamlyn Centre, Faculty of Engineering, Imperial College London, Jun. 2019, pp. 29-30.

[28] Jijie Xu et al., "Motion planning for steerable needles in 3D environments with obstacles using rapidly-exploring Random Trees and backchaining," in 2008 IEEE International Conference on Automation Science and Engineering. Arlington, VA: IEEE, Aug. 2008, pp. 41-46.

[29] N. J. van de Berg et al., "The influence of tip shape on bending force during needle insertion," Scientific Reports, vol. 7, no. 1, p. 40477, Mar. 2017.

[30] K. Reed, A. Okamura, and N. Cowan, "Modeling and Control of Needles With Torsional Friction," IEEE Transactions on Biomedical Engineering, vol. 56, no. 12, pp. 2905-2916, Dec. 2009.

[31] C. Fouard et al., "CamiTK: A Modular Framework Integrating Visualization, Image Processing and Biomechanical Modeling," in Soft Tissue Biomechanical Modeling for Computer Assisted Surgery. Berlin, Heidelberg: Springer Berlin Heidelberg, 2012, vol. 11, pp. 323-354, series Title: Studies in Mechanobiology, Tissue Engineering and Biomaterials.

[32] M. Baumann et al., "Prostate biopsy tracking with deformation estimation," Medical Image Analysis, vol. 16, no. 3, pp. 562-576, Apr. 2012.

[33] J. Xu et al., "Planning fireworks trajectories for steerable medical needles to reduce patient trauma," in 2009 IEEE/RSJ International Conference on Intelligent Robots and Systems. St. Louis, MO, USA: IEEE, Oct. 2009, pp. 4517-4522.

[34] T. K. Adebar and A. M. Okamura, "Recursive estimation of needle pose for control of 3D-ultrasound-guided robotic needle steering," in 2014 IEEE/RSJ International Conference on Intelligent Robots and Systems. Chicago, IL, USA: IEEE, Sep. 2014, pp. 4303-4308.

[35] A. Krupa, "A new duty-cycling approach for 3D needle steering allowing the use of the classical visual servoing framework for targeting tasks," in 5th IEEE RAS/EMBS International Conference on Biomedical Robotics and Biomechatronics. Sao Paulo, Brazil: IEEE, Aug. 2014, pp. 301307.
[36] Wen Sun and R. Alterovitz, "Motion planning under uncertainty for medical needle steering using optimization in belief space," in 2014 IEEE/RSJ International Conference on Intelligent Robots and Systems. Chicago, IL, USA: IEEE, Sep. 2014, pp. 1775-1781.

[37] G. J. Vrooijink et al., "Needle path planning and steering in a threedimensional non-static environment using two-dimensional ultrasound images," The International Journal of Robotics Research, vol. 33, no. 10, pp. 1361-1374, Sep. 2014.

[38] M. Khadem et al., "Ultrasound-Guided Model Predictive Control of Needle Steering in Biological Tissue," Journal of Medical Robotics Research, vol. 01, no. 01, p. 1640007, Mar. 2016.

[39] F. Liu et al., "Fast and Adaptive Fractal Tree-Based Path Planning for Programmable Bevel Tip Steerable Needles," IEEE Robotics and Automation Letters, vol. 1, no. 2, pp. 601-608, Jul. 2016.

[40] B. Fallahi et al., "Sliding-based image-guided 3D needle steering in soft tissue," Control Engineering Practice, vol. 63, pp. 34-43, Jun. 2017.

[41] C. Huang and Y. Lei, "A novel model-based path planning method for robot-assisted flexible needle insertion," in 2017 13th IEEE Conference on Automation Science and Engineering (CASE). Xi'an: IEEE, Aug. 2017, pp. 1414-1419. 